\title{
CDISC CDASH Units for Exposure Terminology
}

National Cancer Institute

\section{Source}

National Cancer Institute. CDISC CDASH Units for Exposure Terminology. NCI

Thesaurus. Code C78423.

A terminology subset of the CDISC Study Data Tabulation Model (SDT M) Unit codelist created for Clinical Data Acquisition Standards Harmonization (CDASH) Units for Exposure terminology. 